\title{
Interacting neutrino gas in dense nuclear matter
}

\author{
Ubiratãn J. Furtado* \\ Departamento de Física - Universidade Federal de Santa Catarina \\ E-mail: ubiratan.furtado@pgfsc.ufsc.br
}

\section{José R. Marinelli}

Departamento de Física - Universidade Federal de Santa Catarina

E-mail: ricardoefsc.ufsc.br

\begin{abstract}
Relativistic models for the many-body system may have applications in nuclear astrophysics, as neutron stars, and also in finite nuclei. In this paper we are interested in analyzing the importance of neutrinos and its interactions within a dense nuclear matter through such a model. We calculate the energy per particle for a many-body system using the mean-field approach, where the meson fields are replaced by their expectation values. The model parameters can be adjusted to agree with results for nuclear matter and astrophysical observations. We start with a Lagrangian density including nucleons, mesons, electrons and neutrinos. After solving the corresponding equations, we use the energy-momentum tensor to calculate the energy density and pressure, as well as neutrino cross sections. Values for the coupling parameters in the model are taken from previous work.
\end{abstract}

XXXIV edition of the Brazilian Workshop on Nuclear Physics

5-10 June 2011

Foz de Iguaçu, Parana state, Brasil

${ }^{*}$ Speaker. 


\section{Introduction}

Relativistic mean-field models have become an standard approach to describe nuclear matter at different density regimes. The model Lagrangian density where nucleons interact through the exchange of scalar, vector and isovector mesons has been widely used for that purpose. Here, our interest is turned to the behavior of the neutrinos inside the hadronic matter, composed by the nucleons and mesons. In particular, we want to investigate the role of the weak force on the neutrino distribution in that system. Effects of a nonzero temperature are considered in our investigation. Only the weak neutral processes are included in our calculation, although the charged weak ones can be incorporated in our results. Also, we obtain the mean-free path for the neutrinos and its dependence with the hadronic density and temperature are presented. For the coupling parameters of the model Lagrangian, we take the values from [5]. However, the extension to other model parametrizations can be easily done and will be considered in a forthcoming paper.

\section{The model Lagrangian}

Considering that our matter consists of nucleons, neutrinos and intermediate bosons, we have the following Lagrangian density ([1], [3]):

$$
\begin{gathered}
\mathscr{L}=\bar{\psi}\left[i \gamma_{\mu} \partial^{\mu}-g_{v} \gamma^{0} V_{0}-\frac{1}{2} g_{\rho} \tau_{3} \gamma^{0} b_{0}-\left(M-g_{s} \phi_{0}\right)-g \frac{\gamma_{0}}{2}\left(c_{V}-c_{A} \gamma^{5}\right) Z_{0}\right] \psi \\
-\frac{1}{2} m_{s}^{2} \phi_{0}^{2}-\frac{1}{2}\left(\frac{\kappa}{3} \phi_{0}^{3}+\frac{\lambda}{12} \phi_{0}^{4}\right)+\frac{1}{2} m_{v}^{2} V_{0}^{2}+\frac{1}{2} m_{\rho}^{2} b_{0}^{2}+\frac{1}{2} M_{Z}^{2} Z_{0}^{2} \\
-g \bar{v}_{L} \frac{\gamma_{0}}{2} Z_{0} v_{L}+i \bar{v} \gamma_{\mu} \partial^{\mu} v
\end{gathered}
$$

where we already have made a mean-field approximation for the bosonic fields(MFT). The nucleon field is $\psi$ and its mass $M$. The neutrino field is $v$ and $v_{L}$ is for left-handed neutrinos. The neutral scalar $\left(\phi_{0}\right)$, neutral vector $\left(V_{0}\right)$ and vector-isovector $\left(b_{0}\right)$ mesons have masses and coupling constants respectively given by $m_{s}$ and $g_{s}, m_{v}$ and $g_{v}$ and $m_{\rho}$ and $g_{\rho}$. The parameters $\kappa$ and $\lambda$ represent higher order scalar meson self-interactions. Within the same approximation, the weak neutral boson $Z_{0}$ is also included in the MFT with the coupling constant $g$ and mass $M_{Z}$. The nucleon isospin is $\tau_{3}=1$ for protons and $\tau_{3}=-1$ for neutrons. Finally, for protons $c_{V}=\left(1-4 \operatorname{sen}^{2}\left(\theta_{w}\right)\right) / 2$ and $c_{A}=1.23 / 2$ and for neutrons $c_{V}=-1 / 2$ and $c_{A}=-1.23 / 2$, where $\theta_{W}$ is the Weinberg angle.

From the above Lagrangian we get the mesonic field equations:

$$
m_{s}^{2} \phi_{0}+\frac{\kappa}{2} \phi_{0}^{2}+\frac{\lambda}{6} \phi_{0}^{3}=g_{s} \rho_{s}, \quad m_{v}^{2} V_{0}=g_{v} \rho_{B} \quad \text { and } \quad m_{\rho}^{2} b_{0}=\frac{1}{2} g_{\rho} \rho_{3},
$$

where the scalar $\left(\rho_{s}\right)$, baryonic $\left(\rho_{B}\right)$ and isoscalar $\left(\rho_{3}\right)$ densities are the source terms for the corresponding fields. For the neutral weak boson we get:

$$
M_{Z}^{2} Z_{0}=\frac{g}{4}\left[\left(1-4 \operatorname{sen}^{2}\left(\theta_{w}\right)\right) \rho_{p}-\rho_{n}\right]+\frac{g}{2} \rho_{v L}-\frac{g}{4}<\psi^{\dagger} c_{A} \gamma_{5} \psi>
$$

with $\rho_{p}$ and $\rho_{n}$ being the proton and neutron densities and $\rho_{\nu L}$ is the left-handed neutrino density. The last term in the above equation contributes very little (if nothing) to our mean-field approach 
and will be disregarded. The energy density and the pressure can be calculated using the energymomentum tensor, after solving the field equations. The energy density, in terms of the nucleon effective mass $M^{*}=M-g_{s} \phi_{0}$, is:

$$
\begin{gathered}
\varepsilon=\sum_{i=p, n} \frac{\gamma}{(2 \pi)^{3}} \int d^{3} k E^{*}\left(\eta_{k i}(T)+\bar{\eta}_{k i}(T)\right)+\frac{1}{(2 \pi)^{3}} \int d^{3} k k\left(\eta_{k v}(T)+\bar{\eta}_{k v}(T)\right) \\
+\frac{1}{2} \frac{g_{v}^{2}}{m_{v}^{2}} \rho_{B}^{2}+\frac{1}{8} \frac{g_{\rho}^{2}}{m_{\rho}^{2}} \rho_{3}^{2}+\frac{1}{2} \frac{m_{s}^{2}}{g_{s}^{2}}\left(M-M^{*}\right)^{2}+\frac{1}{6} \frac{\kappa}{g_{s}^{3}}\left(M-M^{*}\right)^{3}+\frac{1}{24} \frac{\lambda}{g_{s}^{4}}\left(M-M^{*}\right)^{4} \\
+\frac{g}{4}\left(1-4 \operatorname{sen}^{2}\left(\theta_{w}\right)\right) Z_{0} \rho_{p}-\frac{g}{4} Z_{0} \rho_{n}-\frac{1}{2} M_{Z}^{2} Z_{0}^{2}+\frac{g}{2} Z_{0} \rho_{v L} .
\end{gathered}
$$

The pressure is given by:

$$
\begin{aligned}
P & =\frac{1}{3} \frac{\gamma}{(2 \pi)^{3}} \sum_{i=p, n} \int d^{3} k \frac{k^{2}}{E^{*}}\left(\eta_{k i}(T)+\bar{\eta}_{k i}(T)\right)+\frac{1}{3} \frac{1}{(2 \pi)^{3}} \int d^{3} k k\left(\eta_{k v}(T)+\bar{\eta}_{k v}(T)\right) \\
& +\frac{1}{2} \frac{g_{v}^{2}}{m_{v}^{2}} \rho_{B}^{2}+\frac{1}{8} \frac{g_{\rho}^{2}}{m_{\rho}^{2}} \rho_{3}^{2}-\frac{1}{2} \frac{m_{s}^{2}}{g_{s}^{2}}\left(M-M^{*}\right)^{2}-\frac{1}{6} \frac{\kappa}{g_{s}^{3}}\left(M-M^{*}\right)^{3}-\frac{1}{24} \frac{\lambda}{g_{s}^{4}}\left(M-M^{*}\right)^{4}
\end{aligned}
$$

In these equations, $\eta_{k, i}(T)$ is the distribution function for particles and $\bar{\eta}_{k, i}(T)$ is the distribution function for antiparticles:

$$
\eta_{k, i}(T)=\left\{\exp \left[\left(E_{i}-\mu_{i}\right) / K_{b} T\right]+1\right\}^{-1}, \quad \bar{\eta}_{k, i}(T)=\left\{\exp \left[\left(\bar{E}_{i}+\mu_{i}\right) / K_{b} T\right]+1\right\}^{-1}
$$

where $E_{i}$ is the energy of a single particle and $\mu_{i}$ is the chemical potential.

For the nucleons:

$$
E_{N}=E^{*}+\frac{g_{v}^{2}}{m_{v}^{2}} \rho_{B}+\frac{g_{\rho}^{2}}{4 m_{\rho}^{2}} \tau_{3} \rho_{3}+\frac{g}{2} c_{V} Z_{0}, \quad \bar{E}_{N}=E^{*}-\frac{g_{v}^{2}}{m_{v}^{2}} \rho_{B}-\frac{g_{\rho}^{2}}{4 m_{\rho}^{2}} \tau_{3} \rho_{3}-\frac{g}{2} c_{V} Z_{0}
$$

and for the neutrinos:

$$
E_{v}=k+\frac{g}{2} Z_{0}, \quad \bar{E}_{v}=k-\frac{g}{2} Z_{0}
$$

where $\vec{k}$ is the momentum, $\gamma$ is the spin degeneracy (in this case $\gamma=2$ ) and $E^{*}=\sqrt{k^{2}+M^{* 2}}$.

We have also included the electrons in the system as a relativistic free Fermi gas, with the constraint of neutral total charge, $\rho_{p}=\rho_{e}$. The energy density and the pressure for the electrons are:

$$
\begin{gathered}
\varepsilon_{e}=\frac{\gamma}{(2 \pi)^{3}} \int d^{3} k \sqrt{k^{2}+m_{e}^{2}}\left(\eta_{k e}(T)+\bar{\eta}_{k e}(T)\right) \\
P_{e}=\frac{1}{3} \frac{\gamma}{(2 \pi)^{3}} \int d^{3} k \frac{k^{2}}{\sqrt{k^{2}+m_{e}^{2}}}\left(\eta_{k e}(T)+\bar{\eta}_{k e}(T)\right)
\end{gathered}
$$

with

$$
\eta_{k e}(T)=\frac{1}{\exp \left[\left(\sqrt{k^{2}+m_{e}^{2}}-\mu_{e}\right) / K_{b} T\right]+1}, \quad \bar{\eta}_{k e}(T)=\frac{1}{\exp \left[\left(\sqrt{k^{2}+m_{e}^{2}}+\mu_{e}\right) / K_{b} T\right]+1} .
$$




\section{Neutrino mean-free path}

The neutrino mean-free path $\lambda$, is given by:

$$
\lambda=\left(\frac{\sigma}{V}\right)^{-1}
$$

where $\mathrm{V}$ is the volume and the differential cross section for a collision between two particles is:

$$
d \sigma=\frac{<|\mathscr{M}|^{2}>}{F} d Q
$$

where $\left\langle|\mathscr{M}|^{2}>\right.$ is the average over initial spins and the sum over final spins of $|\mathscr{M}|^{2}, \mathscr{M}$ is the scattering amplitude, $F$ is the incident flux and $d Q$ is the Lorentz invariant phase space factor of the final state.

A complete outline of possible processes involving neutrino scattering or absorption is given in [2]. Here we calculate just the elastic scattering cross section of a neutrino by a nucleon or by another lepton. For the scattering $1+2 \longrightarrow 3+4$, where 1 is the incoming neutrino, 3 is the outgoing neutrino, 2 is the incoming nucleon (lepton) and 4 is the outgoing nucleon (lepton), we have [3]:

$$
\begin{gathered}
F=\left|\vec{v}_{1}-\vec{v}_{2}\right| \cdot 2 E_{1}^{*} \cdot 2 E_{2}^{*} \\
d Q=(2 \pi)^{4} \delta^{4}\left(P_{1}+P_{2}-P_{3}-P_{4}\right) \frac{1-\eta_{3}(T)}{2 E_{3}^{*}} \frac{d \vec{p}_{3}}{(2 \pi)^{3}} \frac{1-\eta_{4}(T)}{2 E_{4}^{*}} \frac{d \vec{p}_{4}}{(2 \pi)^{3}} \\
<|\mathscr{M}|^{2}>=\frac{g^{4}}{2 M_{Z}^{4}} E_{1}^{*} E_{2}^{*} E_{3}^{*} E_{4}^{*}\left\{\left(c_{V}+c_{A}\right)^{2}\left(1-v_{2} \cos \left(\theta_{12}\right)\right)\left(1-v_{4} \cos \left(\theta_{34}\right)\right)\right. \\
\left.+\left(c_{V}-c_{A}\right)^{2}\left(1-v_{4} \cos \left(\theta_{14}\right)\right)\left(1-v_{2} \cos \left(\theta_{23}\right)\right)-\frac{M^{* 2}}{E_{2}^{*} E_{4}^{*}}\left(c_{V}^{2}-c_{A}^{2}\right)\left(1-\cos \left(\theta_{13}\right)\right)\right\}
\end{gathered}
$$

where $\eta_{i}(T)$ is the distribution function to the particle $i, v_{i}=\left|\vec{p}_{i}\right| / E_{i}^{*}, P_{i}=\left(E_{i}^{*}, \vec{p}_{i}\right)$ and $\cos \left(\theta_{i j}\right)$ is the angle between $\vec{p}_{i}$ and $\vec{p}_{j}$. We need now to sum over all the momentum $\vec{p}_{2}$, so we multiply $d \sigma$ by $V \eta_{2}(T) d \vec{p}_{2} /(2 \pi)^{3}$. The total cross section is obtained by integrating $d \sigma$ over all the momentum $\vec{p}_{1}, \vec{p}_{2}$ and $\vec{p}_{3}$ :

$$
\begin{gathered}
\frac{\sigma}{V}=\int \frac{d^{3} p_{2}}{(2 \pi)^{3}} \int \frac{d^{3} p_{3}}{(2 \pi)^{3}} \int \frac{d^{3} p_{4}}{(2 \pi)^{3}}(2 \pi)^{4} \delta^{4}\left(P_{1}+P_{2}-P_{3}-P_{4}\right) \frac{1}{\left|\vec{v}_{1}-\vec{v}_{2}\right|} . \\
\eta_{2}(T)\left(1-\eta_{3}(T)\right)\left(1-\eta_{4}(T)\right) \frac{g^{4}}{32 M_{Z}^{4}}\left\{\left(c_{V}+c_{A}\right)^{2}\left(1-v_{2} \cos \left(\theta_{12}\right)\right)\left(1-v_{4} \cos \left(\theta_{34}\right)\right)\right. \\
\left.+\left(c_{V}-c_{A}\right)^{2}\left(1-v_{4} \cos \left(\theta_{14}\right)\right)\left(1-v_{2} \cos \left(\theta_{23}\right)\right)-\frac{M^{* 2}}{E_{2}^{*} E_{4}^{*}}\left(c_{V}^{2}-c_{A}^{2}\right)\left(1-\cos \left(\theta_{13}\right)\right)\right\} .
\end{gathered}
$$

We have calculated the above expression without any low velocity approximation, so in principle our result is suitable for application to any baryonic density regime. In the case of a collision with a electron, we just make the changes $E_{i}^{*} \longrightarrow \sqrt{p_{i}^{2}+m_{e}^{2}}$ and $M^{*} \longrightarrow m_{e}$. Besides, the proper values for $c_{V}$ and $c_{A}$ should be used. 


\section{Results and discussion}

Figure 1 shows the energy per neutrino $\left(\varepsilon_{v} / \rho_{v}\right)$ versus the baryon density for different temperatures, where $\rho_{0}=0.16 \mathrm{fm}^{-3}$ is the nuclear matter equilibrium density and $\varepsilon_{v}$ is the contribution of the neutrinos to the energy:

$$
\varepsilon_{v}=\frac{1}{(2 \pi)^{3}} \int d^{3} k k\left(\eta_{k v}(T)+\bar{\eta}_{k v}(T)\right)+\frac{g}{2} Z_{0} \rho_{v L} .
$$

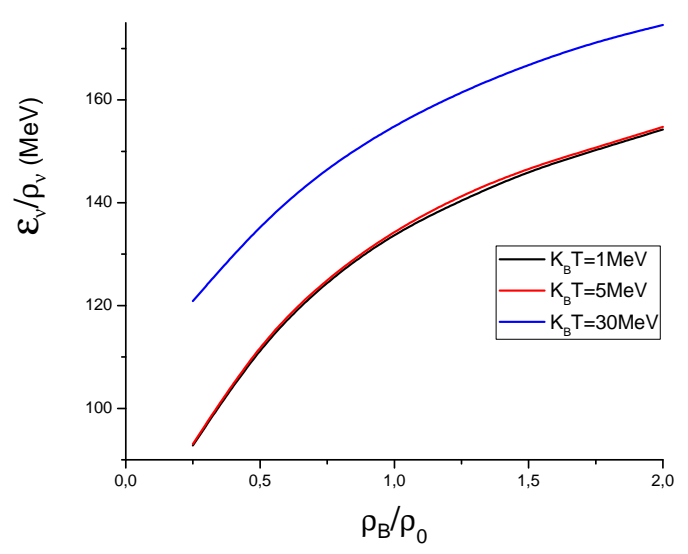

Figure 1: Energy of the neutrinos per neutrino for different temperatures

In figure 2 we see the total energy per nucleon (including the electrons), $\left(\varepsilon+\varepsilon_{e}\right) / \rho_{B}-M$, as a function of the baryon density for different temperatures and the total pressure for different temperatures is shown in figure 3.

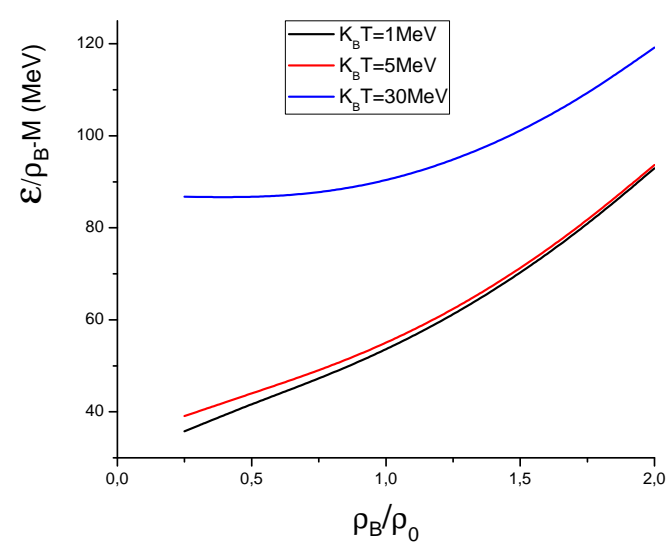

Figure 2: Total energy per nucleon for different temperatures

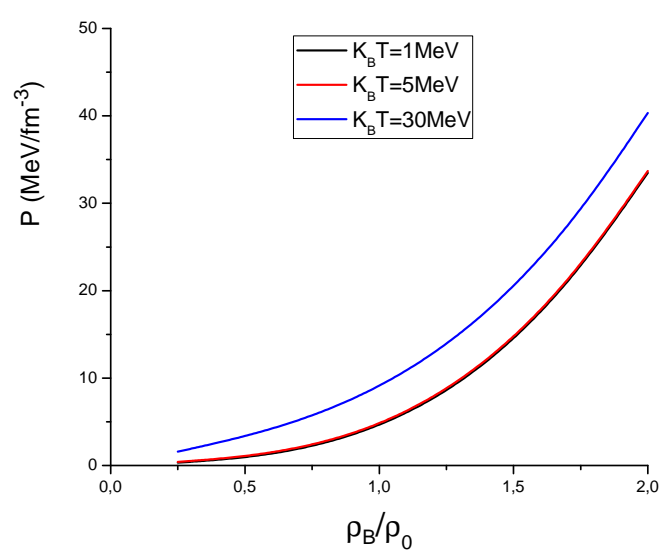

Figure 3: Total pressure for different temperatures 
Finally, figures 4, 5 and 6 show the neutrino mean-free path $\lambda$ as a function of the baryon density for three different temperatures respectively and choosing $E_{v}=\mu_{v}$. The contribution of each kind of particle to the neutrino mean-free path is shown, where $\lambda_{e}, \lambda_{p}$ and $\lambda_{n}$ are the individual contributions due to electrons, protons and neutrons, respectively, and $\lambda_{S}$ is the total mean-free path for the neutrinos.

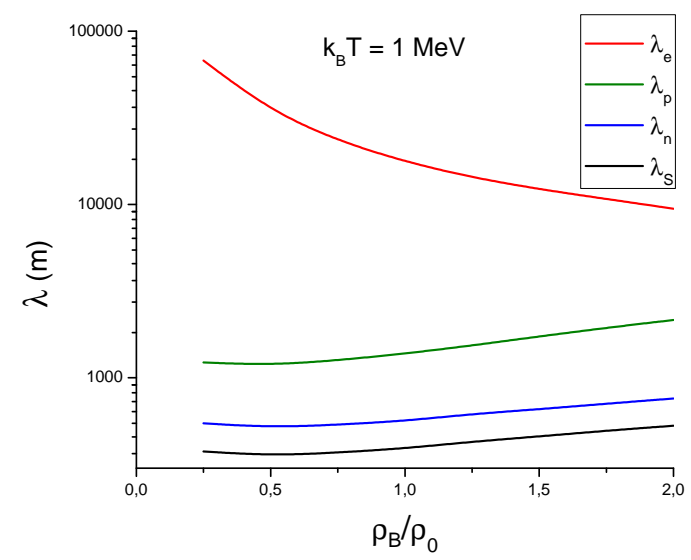

Figure 4: Contribution of each type of particle to the neutrino mean-free path for $k_{B} T=1 \mathrm{MeV}$

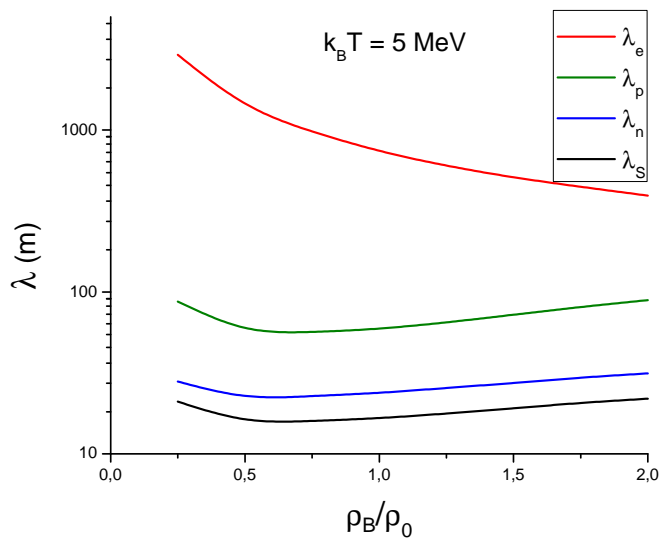

Figure 5: Same as figure 4 for $k_{B} T=5 \mathrm{MeV}$

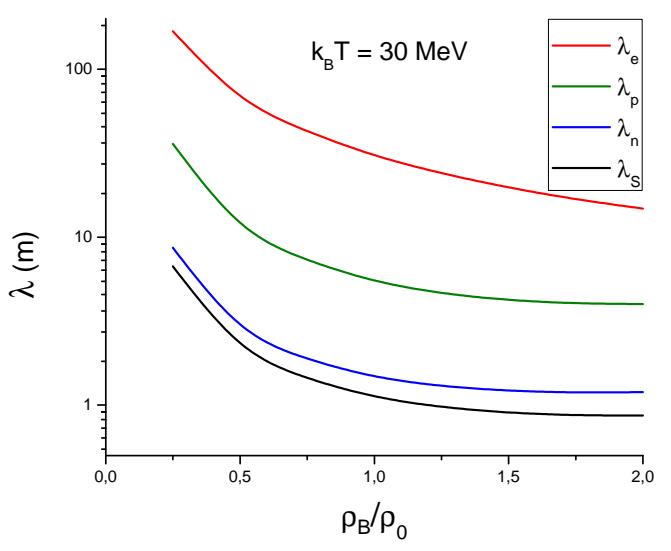

Figure 6: Same as figure 4 for $k_{B} T=30 \mathrm{MeV}$

The results presented are all for $Y_{l}=\left(\rho_{e}+\rho_{v}\right) /\left(\rho_{B}\right)=0.4$, which is the appropriate value for the beginning of the evolution of a nascent neutron star [4]. The solution of our field equations are the obtained with the GM1 parametrization using the compressibility $K=300 \mathrm{MeV}$ and the effective mass $M^{*} / M=0.70$ as constraints [5].

The increase of the energy with the temperature is as expected, as seen in figures 1 and 2. On the other hand, the mean-free path decreases with increasing temperature. This was also expected because since with the increase of the temperature, the number of collisions increases. 
In practice, the inclusion of the neutral weak boson $Z$ did not modify the energies of the nucleons and neutrinos, i. e., the distribution function for each particle type has remained unchanged compared to the case where no weak interactions are taken in to account. This result was expected because the field of the weak boson $Z$, at the mean-field approach, is proportional to the inverse square of its mass, which is of order $10^{2} \mathrm{GeV}$. In addition, the coupling constant for the weak boson $Z$ is an order of magnitude smaller than the meson coupling constants. What remains to be done is to investigate if this behavior keeps the same if we go to higher density regimes, both for the nucleons an for the leptons. This work is now underway and deserves further numerical efforts.

We intend now to include the charged weak bosons in our results, which can make a possible realistic model to study neutron-rich stars. As a promising application, we also intend to solve the relativistic TOV equation [5] for the star, within and without the weak interaction in the model.

\section{References}

[1] J. D. Walecka, Ann. Phys. 83, 491 (1974).

[2] D. G. Yakovlev, A. D. Kaminker, O. Y. Gnedin, and P. Haensel, Phys. Rept. 354, 1 (2001).

[3] Francis Halzen and Alan D. Martin in Quarks and Leptons: An Introductory Course in Modern Particle Physics, John Wiley \& Sons (1984).

[4] Sanjay Reddy, Madappa Prakash, and James M. Lattimer, Phys. Rev. D58, 013009 (1998).

[5] N. K. Glendenning in Compact Stars, Springer, second edition.

\section{ACKNOWLEDGMENTS}

This work was partially supported by CNPq (Brazil). 